\title{
Las economías del APEC frente al cambio climático
}

DOI: $10.32870 /$ mycp.v11i31.302

Ana Bertha Cuevas Tello ${ }^{1}$

Resumen

La declaración de los líderes del Foro de Cooperación Económica Asia-Pacífico después de su reunión anual de 2007 en Sydney, Australia, que versó sobre el cambio climático, la seguridad energética y el desarrollo limpio, fue vista por algunos como una propuesta positiva para combatir el cambio climático sin sacrificar el desarrollo económico; en tanto que otros lo juzgaron como un escrito simbólico por no contener objetivos vinculantes. Este artículo se propone responder las siguientes interrogantes: ¿qué fue lo que motivó al APEC a darle un tratamiento especial al asunto del cambio climático? ¿Cuál es el interés de ofrecer una propuesta alternativa al Protocolo de Kioto, cuando la prioridad del APEC es económica y comercial? ¿Es la declaración de Sydney una propuesta novedosa y efectiva? Y, ¿qué posibilidades tiene la declaración de ser más eficiente que el Protocolo de Kioto?

Palabras clave: APEC, Protocolo de Kyoto, cambio climático, desarrollo limpio, seguridad energética.

\section{Abstract}

The Declaration on Climate Change, Energy Security and Clean Development of the Asia-Pacific Economic Cooperation Forum (APEC) leaders from their annual meeting in Sydney, Australia in 2007, was seen by some as a positive proposal to fight climate change without sacrificing economic development; for others, it was just a symbolic text since it did not include linking objectives. This article aims to answer the following questions: What motivated APEC to give a special treatment to climate change? What is the interest to offer an alternative to the Kyoto Protocol, when APEC's priority is economic and trade related? Is the Sydney Declaration novel and effective? and What possibilities do have to be more efficient than the Kyoto Protocol?

Key words: APEC, Kyoto Protocol, climate change, clean development, energy security.

1. Profesora investigadora del Departamento de Estudios del Pacífico, del Centro Universitario de Ciencias Sociales y Humanidades, de la Universidad de Guadalajara. ORCID

http://orcid.org/0000-0002-4553-8141 


\section{Introducción}

حuando el Foro de Cooperación Económica de Asia Pacífico (APEC) anunció que la agenda de la Cumbre de Líderes en Sydney, Australia, en septiembre de 2007, preveía consensuar estrategias globales para enfrentar el cambio climático, los grupos ecologistas, la sociedad civil, los especialistas y los académicos siguieron con atención esta singular asamblea, que por lo regular se centra en el comercio y el desarrollo económico.

La diferencia entre el objetivo por el cual fue creado el APEC y la inclusión de un asunto ambiental que en primera instancia se contradice con el desarrollo económico, aumentaron las expectativas de la cumbre. No era la primera vez que el APEC consideraba el tema medioambiental, pues se sabe que desde 1993 forma parte de la agenda, sino porque esta organización tiene entre sus miembros a Estados Unidos y Australia, dos de los grandes desertores del Protocolo de Kioto (convenio internacional que tiene como objetivo la reducción de la emisión de gases de efecto invernadero causantes del cambio climático).

Estos dos países han declarado abiertamente su desacuerdo con el objetivo del Protocolo de Kioto por ir en contra de los intereses nacionales de sus respectivas economías y por considerarlo poco funcional al dejar fuera del compromiso obligatorio a países en vías de desarrollo, como China y la India.

Varios factores hicieron que la propuesta del APEC pareciera un documento atrayente, efectivo, prometedor y novedoso: la inminente realidad de que el cambio climático es un asunto global que requiere una respuesta internacional inmediata; el escaso éxito del Protocolo de Kioto; la aparente voluntad política de retomar el compromiso por parte del principal emisor de gases contaminantes (Estados Unidos) y de uno de los países desarrollados que emite mayor dióxido de carbono per cápita (Australia); la inclusión con un papel más activo de la nación con mayor disputa dentro del Protocolo de Kioto (China).

El producto del encuentro de líderes del APEC fue la Declaration on Climate Change, Energy Security and Clean Development, texto visto por algunos como un documento con elementos suficientes para combatir el cambio climático sin sacrificar el desarrollo económico, y, por otros, como un escrito simbólico por no contener objetivos vinculantes. Así, en un clima de incertidumbre surgen una serie de interrogantes: ¿qué fue lo que motivó al APEC a darle un 
tratamiento especial al asunto del cambio climático? ¿Cuál es el interés de ofrecer una propuesta alternativa al Protocolo de Kioto, cuando la prioridad del APEC es económica y comercial? ¿Es la declaración de Sydney una propuesta novedosa y efectiva? ¿Qué posibilidades tiene la declaración de ser más eficiente que el Protocolo de Kioto?

Éstas son algunas de las preguntas que se analizarán en el presente artículo. En el primer apartado se hace un recuento del historial general sobre la posición de las economías del APEC en relación con la conformación de los acuerdos internacionales para disminuir los gases contaminantes. En el segundo apartado se describen las contribuciones de dióxido de carbono que realizaron las economías del APEC en el periodo de 1990-2003. En el tercero, con la información de los apartados anteriores, se presentan las razones por las que el APEC incluyó en la cumbre de 2007 el tema del cambio climático; además, se muestran de manera detallada los temas relevantes de la Declaración de Sydney sobre el cambio climático y se analiza la factibilidad de su aplicación.

\section{La postura de las economías del APEC}

Con excepción de Taiwán y Hong Kong, por no considerarse Estados soberanos, todas las economías del APEC son miembros activos de la Convención Marco de las Naciones Unidas sobre el Cambio Climático (CMNUCC), primer tratado internacional que intenta reducir el calentamiento atmosférico a través de medidas y acuerdos internacionales.

Desde $1992^{2}$ a la fecha, los países del mundo han tenido la plena libertad de firmar y ratificar, si así lo desean, su compromiso ante la CMNUCC. Para 1997 con la ratificación del grueso de los países, se acordó incorporar una adición al tratado en el que se previeran medidas más enérgicas y jurídicamente vinculantes; ${ }^{3}$ así surgió el Protocolo de Kioto.

El objetivo principal del Protocolo de Kioto es que en el periodo 20082012, los países del anexo $1^{4}$ (en su mayoría industrializados) reduzcan el total de sus emisiones de dióxido de carbono a la atmósfera, por lo menos $5.2 \%$ con

2. Fecha en que nace el cMnUcC.

3. Véase http://unfccc.int/portal_espanol/essential_background/items/3336.php.

4. Alemania, Australia, Austria, Bélgica, Bielorrusia, Bulgaria, Canadá, Checoslovaquia, Dinamarca, la Comunidad Económica Europea, España, Estados Unidos, Estonia, Rusia, Finlandia, Francia, Grecia, Holanda, Hungría, Irlanda, Islandia, Italia, Japón, Letonia, Lituania, 
relación con los niveles de 1990, utilizando los mecanismos flexibles ${ }^{5}$ como medios para cumplir el compromiso.

Lo que impedía la entrada en vigor del protocolo era que se condicionaba la ratificación: debían firmarla un número suficiente de países del anexo 1 que fueran los responsables de $55 \%$ de las emisiones de dióxido de carbono. El sistema económico actual, basado en la economía de mercado, se sustenta en la producción y el consumo (generados con base en la quema de combustibles fósiles, lo que permite el crecimiento económico, al tiempo que se eleva la calidad de vida de los habitantes de un país y aumenta la competitividad en el plano internacional). En este sentido, a varios de los países desarrollados les inquietaba, con la firma del convenio, la posibilidad de ver mermado su desarrollo económico, en la disyuntiva de elegir entre reducir las emisiones o mantener la tendencia positiva de crecimiento.

La indecisión de ratificar se sustenta en hechos reales, ya que algunos economistas afirmaban que "los costes globales de la reducción de la contaminación de la Convención de Kioto girarán en torno a un billón de dólares”. ${ }^{6}$

Los países del APEC tuvieron un papel definitorio en la constitución del Protocolo de Kioto, pues la ratificación de alguno de ellos y la ausencia de otros marcaron las características, las fortalezas y las debilidades de cómo hoy se presenta este convenio ante los ojos de la comunidad internacional.

En el cuadro 1 se aprecia que, a excepción de Brunei (recuérdese que sólo los Estados soberanos pueden ser parte de este convenio internacional), todos los países del APEC firmaron su compromiso a los pocos meses de ser creada la CMNUCC, y el grueso de ellos lo ratificaron en los siguientes tres años. Los países que se distinguen por lo tardío de su ratificación son Singapur (1997) y Brunei (2007).

Luxemburgo, Noruega, Nueva Zelanda, Polonia, Portugal, Reino Unido, Rumania, Suecia, Suiza, Turquía y Ucrania.

5. 1. Aplicación conjunta: un país desarrollado invierte en otro país desarrollado, en proyectos de energía limpia. El emisor obtiene certificados para reducir emisiones a un precio menor al que le habría costado en el ámbito nacional. 2. Mecanismos de desarrollo limpio: un país desarrollado invierte en tecnología de desarrollo limpio en un país en vías de desarrollo. El recorte de la contaminación derivado de esta inversión se documenta en un certificado que la compañía puede intercambiar por derechos de emisiones del país de origen. 3. Comercio de emisiones: los países que emitan por debajo del límite impuesto por el protocolo pueden vender su excedente de derechos de emisión a aquellos países que lo excedan.

6. P. Samuelson y William Nordhaus, Economía, España, MacGraw-Hill, 2002. 


\section{Cuadro 1}

Fechas de los compromisos de las economías de APEC ante la CMNUCC y el Protocolo de Kyoto

\begin{tabular}{lrrrr}
\hline Economías & \multicolumn{2}{c}{ CMNUCC } & \multicolumn{2}{c}{ Protocolo de Kyoto } \\
\cline { 2 - 5 } & $\begin{array}{r}\text { Fecha de la } \\
\text { firma }\end{array}$ & $\begin{array}{r}\text { Fecha de la } \\
\text { Ratificación }\end{array}$ & $\begin{array}{c}\text { Fecha de la } \\
\text { firma }\end{array}$ & $\begin{array}{r}\text { Fecha de la } \\
\text { Ratificación }\end{array}$ \\
\hline Australia & $6 / 4 / 1992$ & $12 / 30 / 1994$ & $4 / 29 / 1998$ & NR \\
Brunei & NF & $8 / 7 / 2007$ & $\ldots$ & $\ldots$ \\
Canadá & $6 / 12 / 1992$ & $12 / 4 / 1992$ & $4 / 29 / 1998$ & $12 / 17 / 2002$ \\
Chile & $6 / 13 / 1992$ & $12 / 22 / 1994$ & $6 / 17 / 1998$ & $8 / 26 / 2002$ \\
China & $6 / 11 / 1992$ & $1 / 5 / 1993$ & $5 / 29 / 1998$ & $8 / 30 / 2002$ \\
Corea del Sur & $6 / 13 / 1992$ & $12 / 14 / 1993$ & $9 / 25 / 1998$ & $11 / 8 / 2002$ \\
Estados Unidos2 & $6 / 12 / 1992$ & $10 / 15 / 1992$ & $11 / 12 / 1998$ & NR \\
Filipinas & $6 / 12 / 1992$ & $8 / 2 / 1994$ & $4 / 15 / 1998$ & $11 / 20 / 2003$ \\
Hong Kong & $\ldots$ & $\ldots$ & $\ldots$ & $\ldots$ \\
Indonesia & $6 / 5 / 1992$ & $8 / 23 / 1994$ & $7 / 13 / 1998$ & $12 / 3 / 2004$ \\
Japón & $6 / 13 / 1992$ & $5 / 28 / 1993$ & $4 / 28 / 1998$ & $6 / 4 / 2002$ \\
Malaisia & $6 / 9 / 1993$ & $7 / 13 / 1994$ & $3 / 12 / 1999$ & $9 / 4 / 2002$ \\
México & $6 / 13 / 1992$ & $3 / 11 / 1993$ & $6 / 9 / 1998$ & $9 / 7 / 2000$ \\
Nueva Zelanda & $6 / 4 / 1992$ & $9 / 16 / 1993$ & $5 / 22 / 1998$ & $12 / 19 / 2002$ \\
Papúa Nueva Guinea & $6 / 13 / 1992$ & $3 / 16 / 1993$ & $3 / 2 / 1999$ & $3 / 28 / 2002$ \\
Perú & $6 / 12 / 1992$ & $6 / 7 / 1993$ & $11 / 13 / 1998$ & $9 / 12 / 2002$ \\
Rusia & $6 / 13 / 1992$ & $12 / 28 / 1994$ & $3 / 11 / 1999$ & $11 / 18 / 2004$ \\
Singapur & $6 / 13 / 1992$ & $5 / 29 / 1997$ & $\mathrm{NF}$ & $4 / 12 / 2004$ \\
Tailandia & $6 / 12 / 1992$ & $12 / 28 / 1994$ & $2 / 2 / 1998$ & $8 / 28 / 2002$ \\
Taiwan & $\ldots$ & $\ldots$ & $\ldots$ & $\ldots$ \\
Vietnam & $6 / 11 / 1992$ & $11 / 16 / 1994$ & $12 / 3 / 1998$ & $9 / 25 / 2002$ \\
\hline NR No & & &
\end{tabular}

$\mathrm{NR}=$ No ratificó

$\ldots$ = economías que quedan fueran por no ser estados soberanos.

$\mathrm{NF}=$ No firmó

Fuente: Elaboración propia con datos de World Development 2003, http://www.mct.gov.br/ upd_blob/0014/14197.pdf, http://unfccc.int

Después de que en 1997 en la ciudad de Kioto, Japón, se consensuara la formación del Protocolo de Kioto, las economías del APEC no tardaron más de dos años en firmar el acuerdo; lo difícil fue la ratificación del compromiso. No obstante, 2004 fue un año decisivo en la conformación final del Protocolo de Kioto. Por un lado, Estados Unidos, bajo la administración de George Bush, 
decidió abiertamente negarse a la firma, argumentando daños en su economía doméstica y señalando lo inequitativo del acuerdo, al no obligar a países en vías de desarrollo (como China y la India) a cumplir con el Protocolo. Posterior al retiro de la nación estadounidense, Australia optó por no ratificar el compromiso y anunció lo inútil que resultaba su participación cuando el país más contaminante decidió abandonar el acuerdo y cuando a los países en vías de desarrollo no se les obligaría cumplir con el objetivo. ${ }^{7}$

Por otro lado, en ese mismo año Singapur e Indonesia se adhirieron al Protocolo de Kioto, mientras que con la incorporación de Rusia se cumplió el requisito del Convenio, que exigía la ratificación de una cantidad suficiente de países del anexo 1 que fueran los responsables de $55 \%$ de las emisiones de dióxido de carbono. Así, después de casi siete años de acuerdos y negociaciones, éxitos y adversidades, el 16 de febrero de 2005 se puso en marcha el protocolo. ${ }^{8}$

El primer contratiempo del Protocolo de Kioto fue el anuncio, por parte de Estados Unidos, Australia, Japón, Corea del Sur, China y la India, ${ }^{9}$ de la conformación, en enero de 2006, de la Asociación de Asia-Pacífico sobre Desarrollo Limpio y Cambio Climático (mejor conocida como AP6). A grandes rasgos, la asociación proponía: vencer el calentamiento global mediante la promoción de tecnología no contaminante; responsabilizar al sector privado de reducir las emisiones de gases de efecto invernadero; comprometer tanto a los países desarrollados como a los no desarrollados a perseguir el acuerdo; no imponer a los países miembros obligaciones firmes, y utilizar la energía nuclear para mitigar el flujo de gases contaminantes. ${ }^{10}$

Pese a que fue vista por algunos como un boicot al Protocolo de Kioto, la AP6 resultaba atractiva en el sentido de que en sólo estos seis países se emite casi la mitad de los gases contaminantes del planeta. Sin embargo, después de analizar la propuesta resultaba evidente la baja efectividad y mínima viabilidad de sus propuestas:

7. Mural. Disponible en: http://www.mural/parseo/printpage.asp?categoriaid=24\&.../defaulr. htm.

8. ONU, "El Protocolo de Kyoto: camino hacia la protección del sistema climático planetario". Disponible en: http://www.unfccc.de.

9. Cabe aclarar que pese a que Corea del Sur, Japón, India y China forman parte del AP6, no renunciaron al Protocolo de Kyoto.

10. "La Alianza Asia-Pacífico pretende discutir el próximo miércoles una alternativa al Protocolo de Kyoto", Consumer Eroski, el diario del consumidor, 2006. Disponible en: http://www.consumer.es/web/es/medio_ambiente/2006/01/09/148397.php?print=true. 
1. El Protocolo de Kioto también considera el uso de la tecnología, pero es consciente de los costos que implica a corto y a mediano plazo; por eso propusieron los mecanismos de desarrollo limpio, para que la tecnología limpia más actual la empleen en otros países (desarrollados o en vías de desarrollo), mientras se innova en capital que sea más bondadoso con el medio ambiente.

2. Dejar la responsabilidad de la reducción de gases contaminantes en manos del sector privado es un riesgo alto, pues tratándose de un bien público mundial, los dueños del capital no tienen los incentivos necesarios para disminuir los daños, ya que como Samuelson y Nordhaus mencionan: "las empresas no limitan voluntariamente las emisiones de sustancias químicas nocivas, ni se abstienen siempre de verter residuos tóxicos. Por lo que se considera que el control de la contaminación es una función legítima del Estado". ${ }^{11}$

3. Que el compromiso sea por igual tanto para los gobiernos de los países desarrollados como de los no desarrollados; no obstante, recordemos que el retraso tecnológico entre ellos es amplio y que con base en la teoría de la modernización los países no desarrollados se encuentran en un grado de desarrollo evolutivo que los países desarrollados también pasaron, por lo que las dificultades para hacer frente a este punto son evidentes.

4. La no imposición a los países miembros de obligaciones firmes es un tanto riesgosa, ya que cuando los regímenes internacionales tratan de alcanzar un bien público mundial existe entre los países la posibilidad de comportarse como un free rider. ${ }^{12}$ Así, mayor será la tentación si no existen compromisos vinculantes, lo que aumenta los riesgos.

5. En cuanto a la utilización de la energía nuclear, es evidente que su uso resulta igual o más peligroso que los gases de efecto invernadero. ${ }^{13}$ En suma, a pesar de lo relevante de la AP6, la propuesta no fue mejor que lo que ofrece el Protocolo de Kyoto.

A principios de 2006 Canadá consideró la posibilidad de retirarse del Protocolo, al fundamentar la dificultad para reducir sus emisiones debido al crecimiento de su población, la cual señala es superior a la de Europa. Además, afirmaban

11. P. Samuelson y William Nordhaus, op. cit., p. 45.

12. I. Kaul, I. Grunberg y N. Stern, Bienes públicos mundiales, Nueva York, Oxford, 1999.

13. Eco.Portal.Net. "Cambio climático: Greenpeace crítica a Estados Unidos y Australia por su nuevo intento de hundir el Protocolo de Kyoto", 2006. Disponible en: http://www.ecoportal. net/layout/set/print/content/view/full/55635/. 
que el costo de cumplir con el Protocolo de Kioto sería de 23 a 40 mil millones de dólares para 2010. No obstante haber presentado sus razones, el gobierno canadiense aún no se retira, debido a que le teme al costo político, ya que la mayor parte de la población apoya la intención del convenio internacional. ${ }^{14}$

Pese a que en la actualidad algunos sectores consideran que el Protocolo de Kioto es un convenio débil, no existen los elementos suficientes para considerarlo un fracaso (hay que recordar que el alcance de su objetivo tiene de 2008 a 2012 para su conclusión). En definitiva, las decisiones de algunas de las economías del APEC han sido decisivas en la postura actual del Protocolo de Kioto. Aquí resulta interesante analizar las economías del APEC en relación con la emisión de gases de efecto invernadero; es decir, cómo contribuyen en la generación de este mal global.

\section{Las economías del APEC en relación con la emisión de dióxido de carbono en el planeta}

A finales de 2006 se publicó en Londres el informe Stern, ${ }^{15}$ el cual representa la revisión más amplia sobre los aspectos económicos del cambio climático a escala mundial. El reporte menciona que "el cambio climático afectará los elementos básicos de las personas alrededor del mundo: el acceso al agua, la producción de alimentos, la salud y el ambiente. Cientos de millones de personas podrían sufrir hambre, escasez de agua e inundación de costas a causa del calentamiento del mundo". ${ }^{16}$ La información contenida presentó diversos efectos en la esfera política: por un lado, despertó conciencias y con ella la creación de acciones para revertir los pronósticos (especialmente, en Gran Bretaña, Francia y Alemania), y por otro, fue visto como un documento amenazante hacia el actual manejo doméstico de los programas institucionalizados en contra del cambio climático (Estados Unidos). ${ }^{17}$ El reporte Stern estima que si no se actúa pronto:

14. imac. "Amenaza Canadá con retirarse de Kyoto", 2006. Disponible en: http://www.imacmexico.org/ev_es.php=25225_201\&ID2=DO.

15. Elaborado por Nicholas Stern, académico de impecable reputación y que tuvo el cargo de jefe de economistas de la Banca Mundial.

16. N. Stern (2006) Stern Review on the economics of climate change. Disponible en: http://www. $\mathrm{hm}$-treasury.gov.uk/independent_reviews/stern_review_economics_climate_change/stern_ review_report.cfm.

17. Adnmundo. "El cambio climático: el reporte Stern", consultado el 21 de noviembre de 2006, en: http://www.adnmundo.com/contenidos/reporte_stern_ma_211106.html?PrintPage=1. 
[...] los riesgos y costos en conjunto del cambio climático equivaldrían a perder al menos $5 \%$ del producto interno bruto cada año, ahora y por siempre. Si un amplio rango de riesgo e impactos son tomados en cuenta, la estimación del peligro podría aumentar al $20 \%$ o más. Por el contrario, el costo de actuar para reducir las emisiones de dióxido de carbono para evitar los peores impactos del cambio climático puede ser limitado alrededor del 1\% del PIB por año. ${ }^{18}$

Ante tal pronóstico, es más eficaz tomar acciones económicas ahora que tener que iniciar después, cuando sean más costosas e inevitables. En este sentido, seguir las indicaciones del informe Stern representa una estrategia en pro del crecimiento; ignorarlo terminará por colapsar la economía mundial, y los países no desarrollados serán los más afectados. ${ }^{19}$

En este contexto surge la necesidad de analizar la evolución de la emisión de dióxido de carbono en el planeta utilizando - como si fuera una muestra del comportamiento del mundo-la participación de las economías del APEC, la cual representa la región con mayor crecimiento económico ( $6 \%$ anual en el periodo $1989-2006)^{20}$ y en donde se intercambia $60 \%$ del comercio mundial. La selección de esta región resulta válida, ya que dentro del APEC tenemos a diez economías con desarrollo alto, ocho con desarrollo medio y tres de desarrollo bajo. El análisis se realiza a partir de 1990, fecha en el que el Protocolo de Kioto planteó disminuir 5.2\% de las emisiones, y prosigue sólo hasta 2003 debido a la escasez de datos de una fuente confiable.

Como se puede apreciar en el cuadro 2, la emisión de dióxido de carbono de 1990 a 2003 ha mantenido un crecimiento constante a nivel mundial (1.6\% anual) y mayormente dentro de las economías del APEC, las cuales crecieron en promedio anual casi 2\%. Estados Unidos, China, Rusia y Japón son los mayores contaminantes del grupo; de estos últimos se destaca Rusia por disminuir sus emisiones, en este periodo, 2.2\% anualmente. Papúa Nueva Guinea y Brunei son los países que menor cantidad de gases de efecto invernado emitieron durante este tiempo.

18. N. Stern. Stern Review on the economics of climate change, 2006. Disponible en: http://www. $\mathrm{hm}$-treasury.gov.uk/independent_reviews/stern_review_economics_climate_change/stern_ review_report.cfm.

19. "Cambio climático: actuemos ahora o lo pagaremos después", Protocolo-Foreign Affairs \& Lifestyle, 2006. Disponible en: http://www.protocolo.com.mx/.

20. Russian News \& Information Agency. "Asia-Pacifico es la región más dinámica del planeta desde 1989”, 2007. Disponible en: http://sp.rian.ru/onlinenews/20070903/76230393-print. html. 
Cuadro 2

Emisiones de dióxido de carbono de las economías de APEC y el total mundial, miles de toneladas: 1990-2003

\begin{tabular}{llllllll}
\hline Economías & 1990 & 1991 & 1992 & 1993 & 1994 & 1995 & 1996
\end{tabular}

\begin{tabular}{lrrrrrrr}
\hline Australia & 272,678 & 262,878 & 273,091 & 280,468 & 289,513 & 296,633 & 315,261 \\
Brunei & 5,827 & 5,185 & 5,085 & 5,287 & 5,106 & 5,214 & 5,154 \\
Canadá & 416,500 & 413,118 & 420,273 & 434,812 & 415,992 & 448,060 & 452,676 \\
Chile & 35,391 & 33,639 & 35,159 & 35,742 & 41,244 & 44,277 & 50,505 \\
China & $2,402,790$ & $2,523,280$ & $2,647,260$ & $2,790,890$ & $2,962,920$ & $3,202,840$ & $3,345,060$ \\
Corea del Sur & 241,575 & 265,521 & 290,664 & 317,861 & 343,393 & 374,213 & 408,595 \\
Estados Unidos & $4,825,360$ & $4,835,750$ & $4,811,240$ & $5,093,340$ & $5,191,960$ & $5,191,790$ & $5,296,900$ \\
Rusia & $1,987,280$ & $1,987,280$ & $1,987,280$ & $1,813,440$ & $1,567,930$ & $1,506,000$ & $1,495,680$ \\
Filipinas & 43,983 & 45,722 & 50,269 & 51,202 & 56,086 & 62,896 & 65,878 \\
Hong Kong & 26,227 & 27,595 & 31,712 & 34,923 & 29,883 & 29,919 & 27,532 \\
Indonesia & 149,543 & 178,606 & 201,755 & 219,220 & 223,577 & 225,853 & 271,988 \\
Japón & $1,072,420$ & $1,094,350$ & $1,106,500$ & $1,081,490$ & $1,132,560$ & $1,138,750$ & $1,169,550$ \\
Malasia & 55,370 & 67,788 & 74,451 & 90,556 & 92,800 & 119,186 & 122,445 \\
México & 375,836 & 371,579 & 396,163 & 369,129 & 387,448 & 368,048 & 371,355 \\
Nueva Zelanda & 23,642 & 24,744 & 26,155 & 25,404 & 2,681 & 26,882 & 30,341 \\
PNG & 2,433 & 2,590 & 2,532 & 2,534 & 2,506 & 2,410 & 2,412 \\
Perú & 21,065 & 20,293 & 20,426 & 23,574 & 23,064 & 23,533 & 23,872 \\
Singapur & 45,141 & 44,580 & 45,300 & 53,353 & 64,323 & 46,890 & 53,772 \\
Tailandia & 95,897 & 116,232 & 126,932 & 142,668 & 158,401 & 181,603 & 202,870 \\
Taiwan & $\mathrm{nd}$ & $\mathrm{nd}$ & $\mathrm{nd}$ & $\mathrm{nd}$ & $\mathrm{nd}$ & $\mathrm{nd}$ & nd \\
Vietnam & 21,425 & 21,471 & 22,407 & 22,991 & 26,327 & 29,871 & 35,320 \\
Total Apec & $12,120,383$ & $12,342,201$ & $12,574,654$ & $12,888,884$ & $13,017,714$ & $13,324,868$ & $13,747,166$ \\
Mundo & $20,586,747$ & $21,063,185$ & $21,302,730$ & $21,527,143$ & $21,700,299$ & $22,036,837$ & $22,736,860$ \\
\hline & & & & & &
\end{tabular}

Fuente: elaboración propia con datos de Naciones Unidas (indicadores de los objetivos de desarrollo del Milenio). 


\begin{tabular}{|c|c|c|c|c|c|c|c|c|}
\hline 1997 & 1998 & 1999 & 2000 & 2001 & 2002 & 2003 & Total & $\begin{array}{l}\text { Crec. } \\
\text { Prom. } \\
\text { Anual \% }\end{array}$ \\
\hline 317,010 & 331,391 & 346,263 & 349,599 & 367,858 & 354,152 & 354,731 & $4,411,526$ & 2.0 \\
\hline 5,502 & 3,408 & 4,334 & 5,742 & 5,495 & 5,275 & 4,558 & 71,172 & -1.9 \\
\hline 483,695 & 459,520 & 474,127 & 496,747 & 494,982 & 536,866 & 566,617 & $6,513,985$ & 2.4 \\
\hline 58,171 & 57,502 & 62,618 & 59,539 & 55,228 & 57,251 & 58,591 & 684,857 & 4.0 \\
\hline $3,293,750$ & $3,116,650$ & $2,819,840$ & $2,776,530$ & $3,012,080$ & $3,489,490$ & $4,151,410$ & $42,534,790$ & 4.3 \\
\hline 424,573 & 364,070 & 394,318 & 428,005 & 435,492 & 446,190 & 456,751 & $5,191,221$ & 5.0 \\
\hline $5,563,500$ & $5,513,530$ & $5,608,310$ & $5,803,410$ & $5,757,010$ & $5,805,750$ & $5,799,240$ & $75,097,090$ & 1.4 \\
\hline $1,432,240$ & $1,404,100$ & $1,423,300$ & $1,446,080$ & $1,441,640$ & $1,432,910$ & $1,495,870$ & $22,421,030$ & -2.2 \\
\hline 77,280 & 75,889 & 73,027 & 78,071 & 76,730 & 78,128 & 77,095 & 912,256 & 4.4 \\
\hline 28,891 & 37,344 & 40,063 & 37,552 & 35,931 & 34,705 & 37,865 & 460,142 & 2.9 \\
\hline 279,883 & 208,060 & 248,457 & 276,024 & 288,641 & 326,520 & 295,596 & $3,393,723$ & 5.4 \\
\hline $1,170,120$ & $1,130,600$ & $1,165,720$ & $1,207,980$ & $1,191,390$ & $1,025,480$ & $1,233,640$ & $15,920,550$ & 1.1 \\
\hline 124,472 & 114,057 & 108,097 & 126,599 & 136,017 & 141,529 & 156,680 & $1,530,047$ & 8.3 \\
\hline 388,751 & 390,883 & 392,362 & 396,663 & 408,280 & 399,932 & 416,698 & $5,433,127$ & 0.8 \\
\hline 31,744 & 30,386 & 31,682 & 33,205 & 35,071 & 34,651 & 34,819 & 391,407 & 3.0 \\
\hline 2,455 & 2,351 & 2,431 & 2,431 & 2,482 & 2,482 & 2,515 & 34,564 & 0.3 \\
\hline 26,448 & 26,617 & 28,271 & 27,356 & 26,419 & 25,966 & 26,198 & 343,102 & 1.7 \\
\hline 62,209 & 56,332 & 55,986 & 56,574 & 56,608 & 55,589 & 47,885 & 744,542 & 0.5 \\
\hline 210,227 & 186,517 & 197,110 & 201,714 & 217,266 & 230,827 & 246,372 & $2,514,636$ & 7.5 \\
\hline nd & nd & nd & nd & nd & nd & nd & nd & nd \\
\hline 45,698 & 47,847 & 48,347 & 53,739 & 60,437 & 71,661 & 76,242 & 583,783 & 10.3 \\
\hline $14,026,619$ & $13,557,054$ & $13,524,663$ & $13,863,560$ & $14,105,057$ & $14,555,354$ & $15,539,373$ & $189,187,550$ & 1.9 \\
\hline $22,896,612$ & $22,459,664$ & $22,381,719$ & $22,938,356$ & $23,305,722$ & $23,875,356$ & $25,177,178$ & $313,988,408$ & 1.6 \\
\hline
\end{tabular}


Las economías que generaron mayor crecimiento promedio anual de gases contaminantes son: Vietnam (10.3\%), Malasia (8.3\%), Tailandia (7.5\%), Indonesia (5.4\%), Filipinas y China ( $4.4 \%$ y $4.3 \%$, respectivamente).

Rusia y Brunei disminuyeron anualmente su emisión de gases alrededor de 2\%, mientras que Papúa Nueva Guinea, Singapur y México su crecimiento promedio anual no alcanzó $1 \%$.

\section{Cuadro 3}

Emisión de dióxido de carbono de las economías de APEC: 1990-2003 (crecimiento y contribución porcentual)

\begin{tabular}{|c|c|c|c|c|c|c|c|}
\hline \multirow[t]{2}{*}{ Economías } & \multicolumn{2}{|c|}{$\begin{array}{c}\text { Total de miles de } \\
\text { toneladas }\end{array}$} & \multirow{2}{*}{$\begin{array}{c}\begin{array}{c}\text { Crecimiento } \\
\text { promedio } \\
\text { anual (\%) }\end{array} \\
1990-2003\end{array}$} & \multicolumn{2}{|c|}{$\begin{array}{l}\text { \% con relación } \\
\text { al mundo }\end{array}$} & \multicolumn{2}{|c|}{$\begin{array}{l}\text { Toneladas } \\
\text { per cápita }\end{array}$} \\
\hline & 1990 & 2003 & & 1990 & 2003 & 1990 & 2003 \\
\hline Australia & 272,678 & 354,731 & 2.0 & 1.3 & 1.4 & 16.2 & 18.0 \\
\hline Brunei & 5,827 & 4,558 & -1.9 & 0.0 & 0.0 & 22.7 & 12.7 \\
\hline Canadá & 416,500 & 566,617 & 2.4 & 2.0 & 2.2 & 15.0 & 17.9 \\
\hline Chile & 35,391 & 58,591 & 4.0 & 0.2 & 0.2 & 2.7 & 3.7 \\
\hline China & $2,402,790$ & $4,151,410$ & 4.3 & 11.7 & 16.5 & 2.1 & 3.2 \\
\hline Corea del Sur & 241,575 & 456,751 & 5.0 & 1.2 & 1.8 & 5.6 & 9.6 \\
\hline Estados Unidos2 & $4,825,360$ & $5,799,240$ & 1.4 & 23.4 & 23.0 & 18.9 & 19.8 \\
\hline Filipinas & 43,983 & 77,095 & 4.4 & 0.2 & 0.3 & 0.7 & 1.0 \\
\hline Hong Kong & 26,227 & 37,865 & 2.9 & 0.1 & 0.2 & 4.6 & 5.5 \\
\hline Indonesia & 149,543 & 295,596 & 5.4 & 0.7 & 1.2 & 0.8 & 1.4 \\
\hline Japón & $1,072,420$ & $1,233,640$ & 1.1 & 5.2 & 4.9 & 8.7 & 9.7 \\
\hline Malaisia & 55,370 & 156,680 & 8.3 & 0.3 & 0.6 & 3.1 & 6.4 \\
\hline México & 375,836 & 416,698 & 0.8 & 1.8 & 1.7 & 4.5 & 4.0 \\
\hline Nueva Zelanda & 23,642 & 34,819 & 3.0 & 0.1 & 0.1 & 6.9 & 8.8 \\
\hline Papúa Nueva Guinea & 2,433 & 2,515 & 0.3 & 0.0 & 0.0 & 0.6 & 0.4 \\
\hline Perú & 21,065 & 26,198 & 1.7 & 0.1 & 0.1 & 1.0 & 1.0 \\
\hline Rusia & $1,987,280$ & $1,495,870$ & -2.2 & 9.7 & 5.9 & 13.4 & 10.3 \\
\hline Singapur & 45,141 & 47,885 & 0.5 & 0.2 & 0.2 & 15.0 & 11.3 \\
\hline Tailandia & 95,897 & 246,372 & 7.5 & 0.5 & 1.0 & 1.8 & 3.9 \\
\hline Taiwan & $\mathrm{nd}$ & $\mathrm{nd}$ & $\mathrm{nd}$ & $\mathrm{nd}$ & $\mathrm{nd}$ & 0.0 & 0.0 \\
\hline Vietnam & 21,425 & 76,242 & 10.3 & 0.1 & 0.3 & 0.3 & 0.9 \\
\hline Total APEC & $12,120,383$ & $15,539,373$ & 1.9 & 58.9 & 61.6 & 6.9 & 7.1 \\
\hline Mundo & $20,589,332$ & $25,213,122$ & 1.6 & 100 & 100 & $\ldots$ & $\ldots$ \\
\hline
\end{tabular}

Fuente: elaboración propia con datos de Naciones Unidas (indicadores de los objetivos de desarrollo del Milenio).

* El primer dato es de 1992

$2=$ basado sobre dato de gas natural $\mathrm{n} \mathrm{d}=$ no datos 
En el cuadro 3 se concentra de manera más ordenada la información anterior y se agregan datos que exponen, de manera más completa, la participación del APEC en la emisión de gases en el mundo. Se puede apreciar que en su conjunto, el APEC en 1990 contribuyó con 60\% de las emisiones totales, trece años más tarde la contribución aumentó a $61.6 \%$ del total. Por sí solo, Estados Unidos contribuye, en las dos fechas señaladas, con $23 \%$ de las emisiones del mundo (casi la cuarta parte de ellas). China en 1990 emitía únicamente 12\% del total, empero para 2003 ésta creció a 16.5\%.

A principios de la década de los noventa, Rusia expulsaba $10 \%$ de las emisiones totales del mundo; sin embargo, en 2003 su contribución disminuyó a $6 \%$. Japón, por su parte, en las dos fechas casi contribuyó a la contaminación con la misma cantidad (5\%); no obstante, cabe resaltar la disminución de 3 décimas en el último año analizado. El caso de Canadá resulta excepcional, ya que siendo uno de los países más desarrollados del mundo en la actualidad, su contribución porcentual en la generación de gases mundiales es de poco más de dos puntos.

En 1990 doce economías (Brunei, Chile, Filipinas, Hong Kong, Indonesia, Malasia, Nueva Zelanda, Papúa Nueva Guinea, Perú, Singapur, Tailandia y Vietnam) contribuyeron individualmente en el total mundial no más de $0.7 \%$, aunque para 2003 sólo diez economías mantuvieron esa cifra; el resto (Tailandia e Indonesia) pasaron a 1\%. Es decir, en datos absolutos, más de la mitad de las economías del APEC no aumentan de manera alarmante la emisión de gases de efecto invernadero.

Sin embargo, realizando un análisis en cifras relativas de la emisión de gases contaminantes, es decir, emisión de contaminación per cápita, en el panorama se presentan algunos cambios importantes. Australia, Brunei, Canadá y Singapur que en datos absolutos no contribuían de manera relevante en la emisión total, en términos per cápita sí son grandes contaminantes, pues su aportación en 1990 pasó en promedio las 17 toneladas por persona, las que, con excepción de Brunei y Singapur, incrementaron su volumen en 2003. Se hace la aclaración que las economías que en términos absolutos fueron grandes contaminadoras (Estados Unidos y Rusia), también los son 
en términos relativos; se destaca China por tener una emisión per cápita de sólo dos toneladas, y Japón por no sobrepasar los dos dígitos. Así, Japón y Nueva Zelanda son considerados en términos per cápita como contaminadores de nivel medio.

Gráficamente, la emisión de gases de efecto invernadero presentó durante el periodo la siguiente evolución.

\section{Gráfica 1}

Emisión de dióxido de carbono: 1990-2003

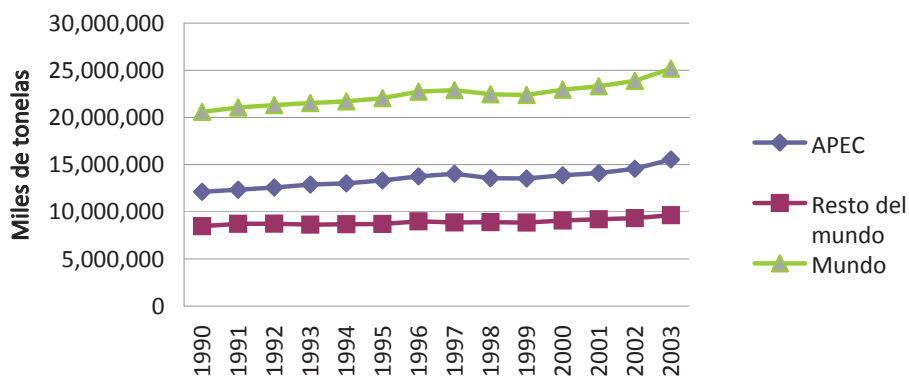

La gráfica 1 muestra la tendencia positiva de la emisión de gases de efecto invernadero en el planeta. Se puede observar la gran proporción con la que contribuyen las 21 economías del APEC en las emisiones totales; es decir, alrededor de 188 países emiten sólo $40 \%$ del total, mientras que únicamente 21 economías son las responsables de 60\% de las emisiones de dióxido de carbono.

Se aprecia que la pendiente de crecimiento de la generación de gases es más prolongada en las economías del APEC que en el resto mundo. Esto habla de que no solamente el bloque de las economías del APEC es una potencia económica y comercial, sino que también es una potencia contaminadora.

Hasta aquí se ha examinado la influencia positiva o negativa que el bloque del APEC puede tener en el funcionamiento y cumplimiento de los objetivos del Protocolo de Kioto, y es evidente que estas economías pueden hacer la diferencia en cuanto a poner freno al cambio climático. En este sentido, resulta interesante analizar qué ofrece la Declaration on Climate Change, Energy Security and Clean Development, y encontrar los verdaderos motivos para crear esta declaración que representa la postura actual del APEC. 


\section{La Declaración Sydney sobre el Cambio Climático, Seguridad Energética y Desarrollo Limpio}

Previo a la Cumbre de Líderes en la ciudad de Sydney, Australia, el APEC lanzó el documento The development costs of the Stern Review finding-implications for building consensus on global strategies for climate change (preparado por The Australian APEC Study Centre, Monash University), el cual es un análisis sobre las propuestas del informe Stern, donde se presentan los elementos para justificar el tema del cambio climático en la mesa de negociación.

El documento señala que existe un consenso mundial que reconoce la necesidad de nuevas estrategias globales para enfrentar el cambio climático, pues "el Protocolo de Kioto ha fallado en alcanzar ese propósito"; al mismo tiempo afirma que es la CMNUCC quien debe conducir este nuevo proyecto. ${ }^{21}$

Después de una introducción loable donde aparecen rasgos de compromiso real, se anuncia que no habrá consenso en la reducción de gases de efecto invernadero, si las estrategias sugeridas restringen el desarrollo económico. Lo anterior se menciona con base en una crítica al informe Stern; se señala que implementar la propuesta de éste sería realmente costoso y que "un consenso solamente se puede construir si permite a cada país desarrollar estrategias sobre el cambio climático que sean capaces de satisfacer las metas económicas nacionales". ${ }^{22}$

En cuanto a la afirmación del informe Stern de que si no se toma una acción fuerte para reducir la emisión de gases ahora, el costo a los países en desarrollo en el largo plazo puede ser muy grande, William Nordhaus tiene un punto de vista diferente: "los costos de una acción temprana podría tener un efecto dañino fuerte en el bienestar de la gente del mundo, particularmente los pobres". ${ }^{23}$

Por último, se concluye que el informe Stern exagera los beneficios económicos y sobreestima los costos económicos de implementar sus recomen-

21. The Australian apec Study Centre, Monash University. "The development costs of the Stern Review finding-implications for building consensus on global strategies for climate change", 2007. Disponible en: http://www.itsglobal.net.

22. The Australian APEC Study Centre, Monash University. "The development costs of the Stern Review finding-implications for building consensus on global strategies for climate change", 2007. Disponible en: http://www.itsglobal.net.

23. Australian APEC Study Centre, Monash University. "The development costs of the Stern Review finding- implications for building consensus on global strategies for climate change, 2007. Disponible en: http://www.itsglobal.net. 
daciones. En este sentido, la Declaración de Sydney parece ser una respuesta alternativa al informe Stern.

La Declaración sobre el Cambio Climático, Seguridad Energética y Desarrollo Limpio inicia con el reconocimiento, por parte de los líderes del APEC, de que el crecimiento económico, la seguridad energética y el cambio climático son desafíos fundamentales e interconectados para la región del APEC. Se menciona que el dinamismo económico del APEC ha reducido la pobreza, mejorado los estándares de vida y elevado el desarrollo social y económico. Pero también se señala que el éxito ha dependido de la oferta energética, la cual al ser usada aumentó los problemas en la calidad del aire y en la emisión de gases de efecto invernadero. Se concluye la introducción con el compromiso de asegurar las necesidades energéticas de la región y afirma su contribución a la reducción de la emisión de gases de efecto invernadero. ${ }^{24}$

La declaración se divide en cuatro apartados: a) Acción internacional futura, b) Apoyo para un acuerdo internacional sobre el cambio climático posterior a 2012, c) Agenda de acción del APEC y d) Los anexos.

En el primer apartado, a grandes rasgos se señala que, basados en los principios de la CMNUCC, los países del APEC creen que de ahora en adelante los acuerdos internacionales sobre el cambio climático, posterior a 2012, deben ser justos y equitativos:

1. Concertación de que todas las economías contribuyan y compartan las metas globales de forma equitativa y efectiva, tanto económica como ambientalmente. 2. Los acuerdos futuros del cambio climático necesitan reflejar diferencias en las condiciones económicas y sociales entre las economías y ser consistentes con el esfuerzo de responsabilidades comunes pero diferenciadas. 3. Para asegurar un esfuerzo global, se apoya los acuerdos flexibles que reconozcan diversos acercamientos y acciones practicas relevantes al cambio climático. 4. Los combustibles fósiles continuarán jugando un papel importante en la región y en las necesidades energéticas globales. La cooperación, incluyendo en la investigación colectiva, el desarrollo, despliegue y transferencia de tecnología de baja y cero emisiones para uso limpio, especialmente carbón, serán esenciales. 5. El manejo sustentable de los bosques y las prácticas en el uso de la tierra juegan un papel clave en el ciclo del carbón, y se necesita tomar medidas posteriores al acuerdo internacional del cambio climático de 2012. 6. En la persecución de cambio climático y las políticas de seguridad energética se debe evitar introducir barreras al comercio

24. APEC. "Sydney apec Leaders' Declaration on Climate Change, Energy Security and Clean Development”, 2007. Disponible en: http://www.apec.org. 
y la inversión. La apertura comercial, la inversión y las políticas ambientales son cruciales para la propagación de los productos de baja emisión, la tecnología y las mejores prácticas. 7. Adaptación a los impactos del cambio climático es una prioridad para las estrategias de desarrollo, las cuales deben ser apoyadas por la comunidad internacional. ${ }^{25}$

En el apartado "Apoyo para un acuerdo internacional sobre el cambio climático posterior 2012", señala grosso modo estar comprometidos con el objetivo global de estabilizar la concentración de gases de efecto invernadero en la atmósfera a niveles que permitan prevenir los peligros en la humanidad "el mundo necesita disminuir, parar y revertir el crecimiento de la emisión de gases de efecto invernadero". ${ }^{26}$ En este sentido, proponen sentar las bases para la configuración de un acuerdo posterior a 2012 con las características mencionadas arriba.

En cuanto al apartado "Agenda de acción del APEC", se menciona reducir la intensidad energética al menos $25 \%$ para 2030 (tomando como año base 2005); abogar por incrementar la cubierta forestal en la región al menos 20 millones de hectáreas de todo tipo de bosques para 2020; establecer una red de tecnología energética para colaborar entre la región y otra de manejo sustentable de los bosques. ${ }^{27}$

Es claro que la Declaración está diseñada para analizar el cambio climático, pues sus principales metas se basan en: 1 . Reducir la intensidad energética 25\% dentro del periodo de 2007-2030, tomando como año base 2005, y 2. Incrementar la cubierta forestal de la región al menos 20 millones de hectáreas para 2020.

Pese a los objetivos anteriores, la Declaración no señala cómo alcanzar estas metas, pues primero reconoce la supremacía de las Naciones Unidas como marco del asunto de cambio climático; luego se invita a todas las economías a responsabilizarse, empero, debido a la diferencia en los grados de desarrollo, las economías están autorizadas a seguir sus propias estrategias, es decir, que cada país fije sus propias metas según su capacidad e intereses.

25. APEC. "Sydney apec Leaders' Declaration on Climate Change, Energy Security and Clean Development", 2007. Disponible en: http://www.apec.org.

26. APEC. "Sydney apec Leaders' Declaration on Climate Change, Energy Security and Clean Development”, 2007. Disponible en: http://www.apec.org.

27. APEC. "Sydney apec Leaders' Declaration on Climate Change, Energy Security and Clean Development”, 2007. Disponible en: http://ww.apec.org. 
En este sentido, ¿quién le va a dar seguimiento a los objetivos el APEC o la CMNUCC? y ¿quién dice qué cantidades son justas para disminuir dependiendo del grado de desarrollo de los países?

En este sentido, es evidente que los objetivos de la Declaración de Sydney tienen el carácter de deseables, no de vinculantes o de obligatorios. Es decir, se está apostando a que las economías del APEC, como responsables de una gran proporción de emisión de gases, acatarán este compromiso de manera moral y solidaria. Este hecho despertó la crítica de ecologistas y defensores del medio ambiente, pues consideraron de muy poca seriedad y decepcionante la propuesta de la Declaración.

Ejemplo de ello fue la manifestación de Abigail Jabines (activista de Greenpeace) la cual señaló que "sin objetivos vinculantes para reducir las emisiones de gases contaminantes, la Declaración de Sydney carece de sentido y es irrelevante a este tema". Agregó, "si Howard y Bush quisieran hacer un esfuerzo al tema del clima, ratificarían el Protocolo de Kioto y adoptarían soluciones reales"; concluyó acusando a estos dos mandatarios de intentar sabotear el Protocolo de Kioto. ${ }^{28}$

Por su parte, Hugh Outhred, especialista energético de la universidad australiana de New South Wales, declaró "que los líderes políticos parecen estar muy preocupados por el clima cuando en realidad hacen muy poco". ${ }^{29}$ Mientras que Catherine Fitzpatrick, activista por la energía de Greenpeace, mencionó "la Declaración de Sydney es solamente una distracción de Sydney de la acción real contra el cambio climático". ${ }^{30}$

28. Emol. "Ambientalistas dicen que declaración de APEC sobre el clima es un gesto vacío", 2007. Disponible en: http://www.emol.com/noticias/internacional/detalle/detallenoticias. asp?idnoticia $=274933$.

LaJornada (2007), Decepciona a ambientalistas acuerdo logrado en el apec sobre el calentamientoglobal.Disponibleen:http://www.jornada.unam.mx/ultimas/2007/09/08decepcionaa-ambientalistas-acuerdo-al

29. Emol. "Ambientalistas dicen que declaración de APEC sobre el clima es un gesto vacío", 2007. Disponible en: http://www.emol.com/noticias/internacional/detalle/detallenoticias. asp?idnoticia $=274933 \#$.

La Jornada, "Decepciona a ambientalistas acuerdo logrado en APEC sobre el calentamiento global”, 2007. Disponible en: http://www.jornada.unam.mx/ultimas/2007/09/08decepcionaa-ambientalistas-acuerdo-al.

30. Hamid Jalil. "Líderes de la región Asia-Pacífico pactó cambio climático en cumbre", 2007. Disponible en: http://Ita.today.reuters.com/misc/. 


\section{Conclusión}

Evidentemente, con base en lo analizado, la Declaración de Sydney prometió mucho y presentó poco, es decir, parece más bien un concentrado de todas las propuestas que les gustaría que tuviera el Protocolo de Kioto (responsabilizar a todos los países; no tener objetivos vinculantes; que las reducciones se realicen conforme el país pueda, no como se le imponga; que las medidas de reducción no pongan en riesgo el desarrollo económico de los países). Asimismo, la Declaración es como una respuesta, con elementos propios, al informe Stern. Y por último, parece ser una estrategia para intentar verse, ante la comunidad internacional, como colaboradores en la reducción de las emisiones de gases de efecto invernadero.

Por otro lado, el planeta presenta un serio problema ambiental. El cambio climático amenaza no sólo con desastres naturales, sino con severas crisis alimenticias, enormes desafíos a la salud y grandes problemas sociales. Todo esto repercutiría, en un futuro no muy lejano, en desequilibrios económicos mundiales, producto de los altos costos financieros, necesarios para hacer frente a la situación.

Las economías del APEC, además de ser potencias económicas y comerciales, son también grandes contaminadores, pues generan más de $60 \%$ de los gases de efecto invernadero que se producen en el planeta. Al mismo tiempo, el ritmo de crecimiento anual de emisiones contaminantes del APEC aumenta más rápidamente que el del resto del mundo. Estos datos muestran, por un lado, el relevante papel que tiene este foro regional en el deterioro ambiental, y por el otro, las grandes posibilidades que poseen, si así lo deciden, como parte de la solución al problema.

Algunas de las economías del APEC son las responsables directas de lo que es hoy, fuerte o débil, el primer acuerdo internacional (con objetivos vinculantes) que hace frente al cambio climático, es decir, el Protocolo de Kioto. En este sentido, el APEC ha fungido como pieza clave dentro de la conformación, la estructuración y la funcionalidad del protocolo, ya que la renuencia de alguna de ellas a ratificar el acuerdo y la revalidación del compromiso por parte de otras, más la disponibilidad de cooperar o no con los objetivos, han armado el mecanismo de acción de lo que actualmente es el Protocolo de Kioto.

Aunque en este artículo no se enumeran los elementos necesarios para medir la efectividad del Protocolo de Kioto, sí hay datos, conforme a las cifras analizadas, que indican que las altas tasas de emisión de gases de efecto inverna- 
dero que se genera en el mundo siguen en constante aumento. En este sentido, la vulnerabilidad del medio ambiente se agudiza y la tranquilidad social se aleja.

Bajo este clima de incertidumbre, en septiembre de 2007, el APEC anunció que en la agenda del encuentro de líderes de ese mismo año los representantes de gobierno se enfocarían en conformar un consenso de estrategias globales para hacer frente al cambio climático. Los grupos sociales que le dan seguimiento al tema tuvieron como respuesta una expectativa esperanzadora. En primer lugar, porque este grupo de economías son las grandes contaminadoras del planeta; en segundo, porque la comunidad internacional siente que es deber del contaminador hacer frente a la solución, y en tercero, porque el grueso de las economías del APEC cuenta con la solvencia económica suficiente para enfrentar los costos que se requieren.

El producto del encuentro del APEC, la Declaration on Climate Change, Energy Security and Clean Development, resultó más un texto compuesto con los elementos que les gustaría a estas importantes economías que el Protocolo de Kioto presentara, que un documento propositivo. Esto debido a que carece de objetivos vinculantes y no presenta datos novedosos. Sobre esto, algunos ambientalistas arguyeron que el documento era poco serio y muy decepcionante.

En este sentido, con base en los elementos analizados, la Declaración de Sydney parece: 1. Una justificación ante la sociedad internacional por el daño ambiental que generan; 2. Que intenta dar la impresión ante los reflectores mundiales de que están haciendo algo en relación con el cambio climático; 3. Un intento por desmeritar al Protocolo de Kioto, y 4. Una manera de pretender proteger el medio ambiente sin dejar de privilegiar al sistema económico. my 